Relations industrielles

Industrial Relations

\title{
La Cour d'appel a-t-elle ouvert de nouveaux horizons en matière de congédiement pour activités syndicales
}

\section{André C. Côté et Marcel Morin}

Volume 29, numéro 4, 1974

URI : https://id.erudit.org/iderudit/028562ar

DOI : https://doi.org/10.7202/028562ar

Aller au sommaire du numéro

Éditeur(s)

Département des relations industrielles de l'Université Laval

ISSN

0034-379X (imprimé)

1703-8138 (numérique)

Découvrir la revue

Citer cet article

Côté, A. C. \& Morin, M. (1974). La Cour d'appel a-t-elle ouvert de nouveaux horizons en matière de congédiement pour activités syndicales. Relations industrielles / Industrial Relations, 29(4), 875-881.

https://doi.org/10.7202/028562ar

Tous droits réservés (C) Département des relations industrielles de l'Université Laval, 1974
Ce document est protégé par la loi sur le droit d'auteur. L'utilisation des services d'Érudit (y compris la reproduction) est assujettie à sa politique d'utilisation que vous pouvez consulter en ligne.

https://apropos.erudit.org/fr/usagers/politique-dutilisation/ 


\title{
LA COUR D'APPEL A-T-ELLE OUVERT DE NOUVEAUX HORIZONS EN MATIÈRE DE CONGÉDIEMENT POUR ACTIVITÉS SYNDICALES ?
}

\author{
ANDRÉ C. CôtÉ \\ MARCEL MORIN
}

Réitérant les grands principes qui ont présidé à l'adoption des articles 14 et ss. C.T. la Cour d'appel souligne que ces articles créent un régime juridique tout à fait particulier et exorbitant du droit commun et consacre l'élargissement des notions de 'salariê' et de 'congédiement' pour activités syndicales.

Depuis déjà quinze ans que nous les retrouvons dans notre droit et malgré l'abondante jurisprudence dont ils ont été l'objet, les dispositions du Code du travail relatives au congédiement pour activités syndicales (arts. 14-16, C.T.) suscitent encore quant à leur application et à leur interprétation des problèmes et des débats aussi fréquents que fondamentaux.

Il y a déjà quelques mois, dans l'affaire United Last Co. Ltd. vs. Le Tribunal du travail ${ }^{1}$, la Cour d'appel confirmait la décision de la Cour supérieure ${ }^{2}$ de refuser l'émission d'un bref d'évocation au profit de l'employeur-requérant.

Les notes du juge Gagnon auxquelles ont souscrit unanimement les juges Montgomery et Turgeon, soulèvent quelques points fort intéressants et démontrent un effort de sensibilisation à l'économie générale du Code du travail et au contexte particulier dans lequel employeurs et employés évoluent qui se retrouve hélas trop rarement chez les magistrats de nos tribunaux de Droit commun.

\section{LES FAITS}

A l'emploi de l'appelante depuis seize ans, Adamowicz reçoit de son employeur le 12 décembre 1969 un avis de mise à pied motivé par

1 (1973) R.D.T. 430.

2 (1973) R.D.T. 423, (décision du juge Jean-Paul Bergeron). De larges extraits de cette décision sont cités dans l'article d'André Roy, Congédiement pour activités syndicales-évolution des décisions, (1974) 29 Relations industrielles, p. 366 aux pp. 394 à 397.

* CÔTÉ, A.C., Professeur, Faculté de droit, Université Laval.

** MORIN, M., Professeur, Faculté de droit, Université Laval. 
un manque d'ouvrage. L'avis mentionne: «Nous vous téléphonerons lorsque l'ouvrage reprendra».

Informé par la suite que plusieurs compagnons de travail mis à pied en même temps que lui avaient été rappelés, Adamowicz se rend à l'usine le 9 février 1970 accompagné de deux autres employés afin de s'enquérir de la date approximative de leur retour au travail. La réception fut plutôt froide : on l'informa que la compagnie n'avait plus l'intention de le rappeler. Des dires mêmes de l'employeur, cette attitude s'expliquait par le fait qu'Adamowicz était un fauteur de troubles et parlait trop de syndicalisme.

En effet, ce dernier avait signé une carte d'adhésion le 5 décembre 1969 et avait fait campagne pour le syndicat.

C'est à la suite de ces révélations qu'Adamowicz logea auprès du commissaire-enquêteur en chef une plainte de congédiement pour activités syndicales le 12 février 1970.

\section{LES ARGUMENTS DE L'APPELANTE}

Le commissaire-enquêteur ayant fait droit à la plainte et le Tribunal du travail ayant entériné cette décision en refusant la permsision d'appeler, la compagnie s'est pourvue en Cour supérieure par voie de bref d'évocation pour le motif que le commissaire-enquêteur et le Tribunal du travail avaient excédé leur juridiction.

L'argument principal de la compagnie tant devant la Cour supérieure que devant la Cour d'appel est résumé par le juge Gagnon en ces mots :

«La mise à pied du 12 décembre 1969 a mis fin au contrat de travail d'Adamowicz. À partir de ce moment, ce dernier n'était plus un salarié au sens de l'article $1(\mathrm{~m})$ C.T., parce que les deux conditions essentielles de l'état de salarié, le travail et la rémunération n'existaient plus. Par voie de conséquence, Adamowicz n'était pas non plus un salarié au sens des articles 14,15 et 16 C.T. de sorte que le commissaire-enquêteur n'avait pas juridiction pour entendre sa plainte, logée tardivement le 12 février 1970. Selon l'appelante, il y a effectivement eu congédiement mais il s'est réalisé dès le 12 décembre 19683

Parmi les autres prétentions de la compagnie notons l'affirmation que : «...si hypothétiquement, un recours pouvait être exercé en vertu de l'article 13 C.T., ou encore du Code civil, celui des articles 14, 15 et 16 C.T., n'était pas ouvert ${ }^{4}$ et que «...le commissaire-enquêteur aurait créé de toutes pièces un lien contractuel inexistant et qu'il a ainsi outrepassé sa juridiction ${ }^{5}$

\footnotetext{
3 Id., p. 432.

4 lbid.,

5 Ibid.,
} 


\section{LA DÉCISION DE LA COUR SUPÉRIEURE}

La décision de la Cour supérieure ne fait que réitérer l'énoncé de certains principes fondamentaux du droit administratif. En particulier le juge Bergeron définit ainsi sa juridiction :

«Il faut d'abord souligner que l'évocation n'est pas un appel de décisions rendues aux termes du Code du travail, qui possède tout son mécanisme propre pour le règlement des conflits survenant dans le monde des relations du travail. Ce code possède au surplus, une clause privative qui réduit davantage le droit d'intervention de la Cour supérieure, pour ne laisser à cette dernière que son droit de regard et de surveillance en cas d'absence ou d'excès de juridiction. $\gg 6$

Et plus loin il ajoute :

«Que le commissaire-enquêteur et le tribunal aient erré ou non dans l'interprétation de ce que constituent un 'salarié' et un 'congédiement', vocables utilisés par la loi qu'ils sont chargés d'administrer, il demeure que le commissaire-enquêteur et le tribunal font partie essentielle du mécanisme prévu par le Code du travail pour régler les conflits de cette nature, et la Cour supérieure ne peut se substituer à ces organismes pour remplacer leurs décisions par les siennes pour le seul motif, en somme, qu'elle ne partagerait pas leur opinion en matière d'interprétation de ces mêmes termes. » 7

\section{LA DÉCISION DE LA COUR D'APPEL}

Heureusement, la Cour d'appel s'est montrée beaucoup moins laconique dans ses considérations et nous a laissé quelques réflexions fort intéressantes sur la nature et la portée des articles 14 et suivants du Code du travail.

Le juge Gagnon se prononce en fait sur le fond du litige et arrive à la conclusion qu'il n'y a pas eu erreur de droit de la part du commissaire-enquêteur et du Tribunal du travail :

« À mon avis, non seulement Adamowicz était un salarié lorsqu'il a été mis à pied, mais il a gardé cette qualité, pour les fins particulières et spéciales de l'application des articles 14,15 et 16 C.T., jusqu'au moment où l'appelante lui a fait savoir qu'elle transformait sa décision de le suspendre temporairement à cause d'un manque d'ouvrage en un congédiement parce qu'il causait du trouble et qu'il parlait trop de syndicat ... $>8$

Cette décision découle de l'analyse que le juge fait du régime de «sécurité d'emploi spéciale » institué par ces articles du Code du travail qu'il
6 Id., p. 427.
7 Id., p. 428.
8 Id., p. 436. 
qualifie de "complet par lui-même » ${ }^{9}$. Ces dispositions, affirme-t-il, «sont tout à fait exorbitantes du droit commun $\gg 10$.

Identifiant le problème comme étant strictement un problème de procédure - la plainte avait-elle été logée en dehors des délais de rigueur de l'article 15 C.T. ? - le tribunal en arrive à circonscrire le sens du terme «salarié » dans le cadre des art. 14-16 C.T. et ensuite à considérer le non-rappel au travail comme équivalent à un congédiement.

A l'argument du requérant à l'effet qu'Adamowicz n'était plus un «salarié » au sens de l'art. $1 \mathrm{~m} \mathrm{C.T.} \mathrm{au} \mathrm{moment} \mathrm{où} \mathrm{il} \mathrm{logea} \mathrm{sa} \mathrm{plainte,} \mathrm{le}$ tribunal répond en affirmant que :

«...le salarié qui a été congédié et qui par conséquent ne travaille plus pour son patron et n'en reçoit plus de rémunération demeure un 'salarié' pour exercer les droits que lui confèrent l'article 15 de porter plainte de son congédiement $\gg 11$

Cette allégation ressort du texte même de l'article 14 C.T. qui dit que ce recours est ouvert «lorsqu'un salarié est congédié, suspendu ou déplacé...» Il faut évidemment que cette plainte soit logée dans les délais prévus à l'article 15 C.T., et l'expiration de ce délai entraine déchéance du droit.

Dans l'affaire qui nous occupe, la question de savoir si Adamowicz était un «salarié » était intimement liée à la réponse qu'on allait apporter à la seconde question : est-ce que son congédiement avait eu lieu à la date de sa mise à pied, auquel cas, même en admettant que cette mise à pied eût été équivalente à un congédiement, sa plainte aurait été tardive, ou encore est-ce que c'est la date de son non-rappel au travail qui servait de point de départ au calcul du délai prévu à l'article 15 ?

Quant à la notion de «congédiement », le juge Gagnon énonce comme position de principe que :

« les termes 'congédiés' et 'congédiement' doivent donc recevoir une interprétation assez large pour que ne soit pas frustrée l'intention des législateurs et qui, à mon avis, devrait couvrir toutes les formes de terminaisons d'emploi motivées par des activités syndicales 》. 12

Une telle prise de position consacrant l'élargissement de la notion de congédiement était certes des plus souhaitable. Elle situe les articles 14 et suivants du Code dans leur véritable contexte et perspective. Que l'on songe, comme le souligne André Roy dans son article précité ${ }^{13}$ que la Commission des Relations de Travail avait adopté à cet égard une position des plus légaliste.

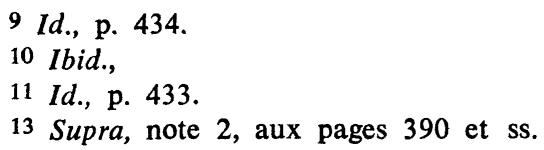


Dans les circonstances, le non-rappel au travail d'Adamowicz constituait un congédiement pour activités syndicales parce que s'il en eut été autrement, l'employeur aurait pu réaliser indirectement ce que la loi lui défendait de faire directement et ce, nonobstant la bonne ou mauvaise foi qui l'animait au moment de la mise à pied :

« Peu importe à mon avis que l'appelante ait décidé, dès décembre 1969, de se débarrasser définitivement d'Adamowicz. Si tel est le cas, je ne suis pas disposé à la faire profiter de sa ruse, ni à pénaliser Adamowicz parce qu'il a eu foi à sa parole. Dans le cas hypothétique où la mise à pied aurait été faite de bonne foi et pour le motif qu'elle a alors indiqué j'irais jusqu'a dire que la décision de l'appelante de ne pas rappeler Adamowicz à cause de l'exercice, avant sa mise à pied, d'un droit que lui reconnaissait le Code du travail, constituerait un 'congédiement' 》 14

L'employé non couvert par une convention collective doit, nous dit le juge Gagnon, généralement « rechercher sa sécurité d'emploi » ${ }^{15}$ (sic) dans le Code civil. Même s'il y a convention collective, dès que les éléments de la présomption de l'article 16 C.T. sont établis à la satisfaction du commissaire-enquêteur, l'employeur

«... ne pourra se retrancher derrière le Code civil et justifier le renvoi de son employé en disant, par exemple, que ce dernier avait été engagé pour une période indéterminée, qu'il était payé à la semaine et qu'il a reçu l'avis d'une semaine. Il devra prouver une cause juste et suffisante de renvoi. $\gg 16$

Il est pour le moins étonnant de voir que le juge Gagnon semble confondre la sécurité d'emploi avec le délai congé. L'utilisation paradoxale de l'expression 'sécurité d'emploi' dans ce contexte constitue certainement un euphémisme.

Le juge Gagnon fait alors quelques affirmations fort significatives :

«On voit donc, dit-il, que ces dispositions débordent les cadres du contrat individuel de travail et qu'elles accordent au salarié congédié pour activités syndicales une sécurité d'emploi qui ne trouve pas sa source dans le lCode civil et qui en rendent même certaines dispositions inapplicables $\gg 17$

14 (1973) R.D.T. 430 à la p. 437. Les italiques sont de nous.

15 (1973) R.D.T. 430 à la p. 434.

16 Id., p. 435; soulignons au passage le court commentaire de Me Gagnon, Lebel et Verge, Chroniques régulières, (1974) $34 R$. du B. p. 250 où les auteurs manifestent leur espoir «que cette notion de cause juste et suffisante » à l'égard de laquelle les juridictions du travail paraissent encore réticentes dans l'application de l'article 16 finisse par être éclaircie sinon par eux, du moins par les tribunaux supérieurs 》.

17 Ibid., Les italiques sont de nous. 
Dès que la présomption de l'article 16 a été établie, le congédiement d'un employé est couvert par un régime juridique tout à fait particulier. Cette particularité se manifeste autant par le fardeau de la preuve qui incombe à l'employeur que par la sanction d'un tel congédiement.

Sur la portée de cette double sanction du congédiement, la réintégration et l'indemnité, le juge Gagnon ajoute :

«... Le commissaire-enquêteur peut ordonner à l'employeur de réintégrer le salarié dans son emploi. Il peut appliquer ces dispositions, je le répète, dans tous les cas, même dans le cas où l'employé n'aurait aucun droit, en vertu de son contrat de travail, de réclamer son réengagement. Le Code du travail confère donc au salarié visé par ces articles un droit spécial à son emploi.

En outre, le commissaire-enquêteur peut ordonner le paiement au salarié du salaire et des autres avantages dont son 'congédiement' l'a privé. Le texte précise qu'il s'agit d'un paiement à titre d'indemnité. C'est en quelque sorte une pénalité imposée à raison de l'acte illégal posé et qui va beaucoup plus loin, malgré les apparences premières, que simplement replacer les parties dans la situation juridique dans laquelle elles se trouvaient avant le congédiement. Sous cet aspect, je dirais que les articles 14,15 et 16 créent entre l'employeur et le salarié une nouvelle situation juridique, qu'ils modifient le contrat individuel de travail et qu'ils donnent au salarié de nouveaux droits, indépendamment du Code civil et même de toute convention collective de travail. $\gg 18$.

On ne peut que se réjouir de voir que la Cour d'appel ait formulé de façon aussi claire le caractère exceptionnel et «sui generis» des protections consacrées par le législateur aux articles 14-16 C.T.

Devant de tels énoncés de principes, n'y aurait-il pas lieu d'explorer plus à fond la possibilité d'obtenir des tribunaux de droit commun des injonctions ordonnant, sous toutes peines que de droit, à ces employeurs refusant de se conformer aux ordonnances de réintégration émises par les commissaires-enquêteurs, de reprendre à leur emploi les employés congédiés pour activités syndicales ? A la lumière de cette décision de la Cour d'appel, ne serait-on pas justifié de penser que la Cour supérieure aurait à faire abstraction, dans le cas de congédiement pour activités syndicales, du principe civiliste voulant qu'un employeur ne peut être forcé de reprendre à son emploi un employé congédié injustement parce que c'est une obligation de faire qui se résoud en dommages-intérêts ? ${ }^{19}$

Les sanctions pénales dont est passible un employeur qui refuse de se conformer à une ordonnance de réintégration sont actuellement si inadé-

18 Id., ceux pp. 435-436; les italiques sont de nous.

19 Dupré Quarries Ltd c. Dupré, (1934) R.C.S. 528; voir à ce sujet P. Verge, «La réintégration forcée du salarié en droit québécois», (1970) 25 Relations industrielles 594. 
quates et insignifiantes ${ }^{20}$ que le législateur dans son récent projet de loi modifiant le Code du travail ${ }^{21}$ s'est senti obligé de rendre considérablement plus lourdes les pénalités contre l'employeur récalcitrant. Malgré cela, l'amende, même très lourde, saura-t-elle assurer le respect de ces ordonnances dans tous les cas ? D'ailleurs le mécanisme d'une plainte pénale est certes un recours assez peu orthodoxe pour assurer le respect d'une ordonnance d'un tribunal et ne saurait compenser la grande difficulté d'accès à un recours qui semble découler beaucoup plus logiquement de celle-ci et qui est destiné par sa nature même à procurer le remède recherché.

En se montrant ouvert à l'émission de telles injonctions, les tribunaux mettraient d'ailleurs fin à une situation fort paradoxale : autant le recours à l'injonction est d'accès facile et fréquent pour forcer un groupe de salariés en grève illégale à retourner au travail, autant il a été restreint, sionn inaccessible, lorsqu'il s'est agit de forcer un employeur à reprendre à son emploi un employé congédié illégalement. ${ }^{22}$

20 Article 126 C.T.; voir à titre d'illustration Daniel Dupuis c. Montreal Hardware, (1971) T.T. 93.

21 Projet de loi no 24 : Loi modifiant le Code du travail et d'autres dispositions législatives, (1ère lecture) 1974, art. 65; cette disposition ajouterait l'article 129a au Code du travail et se lirait comme suit: «L'employeur qui n'exécute pas l'ordonnance de réintégration rendue en vertu de l'article 14 commet une infraction et est passible d'une amende de $\$ 500$. par jour de retard, en sus du paiement de l'indemnité au salarié en vertu de l'article $14 »$.

22 Dans leur ouvrage : Droit du travail en vigueur au Québec, Mes Gagnon, Lebel et Verge soulignent que : «...dans l'état actuel du droit, l'on ne saurait trop espérer obtenir d'une action intentée devant le tribunal de droit commun l'exécution en matière de l'obligation de l'employeur de réintégrer le salarié, en raison, selon la formulation classique, du caractère personnel des prestations qui découlent du contrat de travail et de l'impossibilité d'en forcer l'exécution en nature ». p. 100; voir cependant Syndicat des employés de Montreal Hardware c. Montreal Hardware Mfg. Co. Ltd., (1970) R.D.T. 449 confirmé par (1971) C.A. 592. 